\title{
COVID-19: Wäre eine Impfpflicht rechtlich möglich?
}

Fabian-Simon Frielitz, Isabel Viola Wagner, Denis Martin Schewe, Klara Bothe

\author{
Die COVID-19-Pandemie stellt unser Gesundheitssystem vor beispiellose Heraus- \\ forderungen. Durch Kontaktbeschränkungen, das Tragen von Schutzmasken und weitere \\ Hygienevorgaben, aber auch durch die Impfung soll die Bevölkerung vor einer weiteren \\ Ausbreitung von SARS-CoV-2 geschützt werden. Wie sieht die derzeitige Rechtslage im \\ Zusammenhang mit einer hypothetischen Impflicht aus?
}

Entgegen der wissenschaftlichen und politischen Bestrebung, einen Impfstoff gegen SARS-CoV-2 schnellstmöglich in ausreichender Menge verfügbar zu machen, lehnt ein Teil der Bevölkerung das Impfangebot aufgrund von möglichen Sicherheitsbedenken ab. Auch die jüngsten Entwicklungen der Novellierung des Infektionsschutzgesetzes tragen zur Verunsicherung bei.

\section{Das Infektionsschutzgesetz als Rechtsgrundlage}

Zur möglichst flächendeckenden Immunisierung der Bevölkerung kann unter Verwendung eines geeigneten Impfstoffes hypothetisch eine staatlich verpflichtende Impfung eine mögliche Lösung sein. Eine solche gesetzliche SARS-CoV-2-Impfpflicht setzt voraus, dass der Bund über die sog. Gesetzgebungskompetenz verfügt, um eine bundesweite Regelung zu erlassen. Gemäß Art. 74 Abs. 1 Nr. 19 Grundgesetz (GG) ist der Bund im Rahmen der konkurrierenden Gesetzgebungskompetenz zuständig für „Maßnahmen gegen gemeingefährliche oder übertragbare Krankheiten beim Menschen“. Der Bund hat von dieser Gesetzgebungskompetenz Gebrauch gemacht, indem er das Gesetz zur Verhütung und Bekämpfung von Infektionskrankheiten beim Menschen, das Infektionsschutzgesetz (IfSG) [1], erlassen hat, welches am 19.11.2020 novelliert wurde [2].

Der Zweck des IfSG ist es, als besonderes Gefahrenabwehrrecht der Übertragung von Krankheiten beim Menschen vorzubeugen, Infektionen und Krankheiten frühzeitig zu erkennen und ihre Weiterverbreitung zu verhindern [ $\S 1$ Absatz 1 IfSG]. Das IfSG ermächtigt die zuständigen Behörden zu zahlreichen Maßnahmen, wie etwa Maßnahmen zur Infektionsverhütung nach § 16 IfSG oder Maßnahmen zur Infektionsbekämpfung nach $\S \S 24 \mathrm{ff}$. IfSG. Eine Ermächtigungsgrundlage für eine generelle Impfpflicht der gesamtdeutschen Bevölkerung beinhaltet das IfSG nicht. Allerdings ermöglicht § 20 Abs. 6 IfSG es, dass das Bundesministerium für Gesundheit (BMG) durch Rechtsverordnung festlegen kann, dass im Falle übertragbarer Krank- heiten mit klinisch schweren Verlaufsformen, die die Gefahr einer epidemischen Verbreitung begründen, bedrohte Teile der Bevölkerung an Schutzimpfungen teilzunehmen haben. Eine solche Rechtsverordnung bedarf der Zustimmung des Bundesrates.

Das BMG hat bisher von dieser Kompetenzmöglichkeit, eine allgemeine und flächendeckende Impfpflicht festzulegen, keinen Gebrauch gemacht, sodass es den Ländern nach § 20 Abs. 7 IfSG obliegt, eine entsprechende Rechtsverordnung zu erlassen. Bislang haben auch die Länder keine Rechtsverordnungen vorgelegt, sodass derzeit keine Rechtsgrundlage für eine Impfpflicht im Seuchen- und Infektionsfall der Gesamtbevölkerung besteht. Gleichwohl wäre eine solche Schutzimpfung verpflichtend nach dem IfSG (zumindest für bedrohte Bevölkerungsgruppen) möglich.

In Deutschland besteht Impfpflicht gegen Masern für Kinder und das betreuende Personal wie Lehrer und Kindertagespflegekräfte. Sie ist auf Grundlage des Masernschutzgesetzes (MSG) mit Wirkung zum 01.03.2020 in Kraft getreten [3]. Das Gesetz hat die Eindämmung der Maserninfektion zum Ziel und sieht u. a. vor, dass Kinder, die in einer Kindertagesstätte oder in der erlaubnispflichtigen Kindertagespflege betreut werden, einen ausreichenden Impfschutz oder eine Masernimmunität aufweisen müssen. Eine Ausnahme gilt, wenn sie aufgrund einer medizinischen Kontraindikation nicht geimpft werden können.

Das Bundesverfassungsgericht (BVerfG) hat eine solche Masernimpfpflicht als zulässig erachtet mit der Begründung, dass „Impfungen gegen Masern (...) nicht nur das Individuum gegen die Erkrankung schützen, sondern gleichzeitig die Weiterverbreitung der Krankheit in der Bevölkerung verhindern (...). Auf diese Weise können auch Personen geschützt werden, die aus medizinischen Gründen selbst nicht geimpft werden können, bei denen aber schwere klinische Verläufe bei einer Infektion drohen“" [4]. Ob das BVerfG bei einer potenziellen Impfpflicht gegen das SARS-CoV-2-Virus ebenso argumentieren würde, bliebe abzuwarten. 


\section{Die potenzielle SARS-CoV-2-Impfpflicht}

Art. 2 Abs. 2 S. 1 GG schützt vor staatlichen Eingriffen in das Leben oder den Körper des Grundrechtsträgers und umfasst sowohl die körperliche Unversehrtheit als auch die Integrität und die physische Gesundheit eines jeden einzelnen Menschen. Daraus folgt, dass medizinische Behandlungen grundsätzlich einer Einwilligung des Grundrechtsträgers bedürfen. Demgegenüber hat der Staat aus Art. 2 Abs. 2 GG grundsätzlich die Pflicht, das verfassungsrechtlich garantierte Grundrecht auf Leben und körperliche Unversehrtheit zu wahren und zu schützen. Diese Schutzpflicht steht jedem Einzelnen im Geltungsbereich des GG zu. So haben besonders anfällige Menschen und Personen mit Vorerkrankungen den Anspruch, vor besonders schwerwiegenden Erkrankungen geschützt zu werden. Gefährdet sind neben alten Menschen besonders chronisch kranke und immunsupprimierte Patienten. Dies gilt auch hinsichtlich des SARS-CoV-2-Virus.

Ob das Grundrecht aus Art. 2 Abs. 2 GG verletzt ist, richtet sich danach, ob in den grundrechtlichen Schutzbereich durch eine Impfverpflichtung eingegriffen wird und dieser Eingriff verfassungsrechtlich ungerechtfertigt ist. Ein Grundrechtseingriff liegt vor, wenn eine finale, unmittelbare und gezielte Beeinträchtigung eines Grundrechts bei einem bestimmten Grundrechtsträger erfolgt. Eine vorbeugende Schutzimpfung ist die Injektion eines Impfstoffes, um eine körperliche Abwehr und damit eine Immunisierung herbeizuführen und greift damit in Art. 2 Abs. 2 GG ein. Der Schutzbereich dieses Grundrechts ist nicht erst betroffen, wenn die Impfung im Einzelfall die Gesundheit gefährdende Reaktionen, z. B. in Form von Nebenwirkungen, Kontraindikationen oder Allergien, hervorruft, sondern bereits durch die Injektion eines Impfstoffes selbst.

Ein Grundrechtseingriff ist jedoch verfassungsrechtlich möglich, wenn eine Rechtfertigung besteht und das Grundrecht auf körperliche Unversehrtheit überhaupt einschränkbar ist. Nach Art. 2 Abs. 2 S. 3 GG besteht ein Gesetzesvorbehalt. Dies bedeutet, dass das Grundrecht aus Art. 2 Abs. 2 S. 1 GG aufgrund eines Gesetzes beschränkt werden kann. Das IfSG ist ein solches Gesetz, welches es nach $\S 20$ Abs. 6 IfSG erlaubt, eine Schutzimpfung für bedrohte Teile der Bevölkerung durch Rechtsverordnung anzuordnen, wenn eine übertragbare Krankheit mit klinisch schweren Verlaufsformen auftritt und mit ihrer epidemischen Verbreitung zu rechnen ist.

\footnotetext{
Merke

Nach dem IfSG kann grundsätzlich eine Impfpflicht für eine bestimmbare Personengruppe festlegt werden. Diese Verpflichtung muss als grundrechtlicher Eingriff verhältnismäßig sein. Verhältnismäßig und damit verfassungsrechtlich gerechtfertigt ist ein Eingriff, wenn dieser ein legitimes Ziel verfolgt, geeignet und erfor-
}

derlich, da kein anderes milderes Mittel zur Verfügung steht, und angemessen ist.

Eine potenzielle Schutzimpfungspflicht verfolgt unstreitig ein legitimes Ziel und ist geeignet und erforderlich, die Ausbreitung des SARS-CoV-2-Virus zu verhindern, da derzeit keine anderen und gleich effektiven Mittel zur Verfügung stehen, eine langfristig flächendeckende Eindämmung der Infektion zu erzielen. Maßnahmen wie z. B. vorübergehende häusliche Quarantäne, lokale Schulschließungen, Beschränkungen von Wirtschaftszweigen oder Reisen sind nicht gleich effektiv, da sie zum einen nur kurzzeitig Wirkung entfalten und zum anderen räumlich begrenzt sind. Auch eine natürliche Immunisierung durch die Infizierung mit dem SARS-CoV-2-Virus ist nicht gleich effektiv, da zeitlich nicht vorhersehbar ist, ob und wann eine flächendeckende und lückenlose Herdenimmunität in der Bevölkerung auftritt und dauerhaft anhält. Auch ist unklar, ob eine solche natürliche Herdenimmunität ohne schwerwiegende Krankheitsverläufe möglich ist, ohne das Gesundheitssystem, insbesondere im intensivmedizinischen Versorgungsbereich, an die Belastungsgrenze zu bringen. Durch eine verpflichtende Impfung könnten auch Menschen geschützt werden, die aus medizinischen Gründen selbst nicht geimpft werden können, indem ihr Umfeld eine Immunität entwickelt. Es ist damit eine Abwägung zu treffen zwischen dem Anspruch gegenüber dem Staat, vor besonders risikoreichen und folgenschweren Erkrankungen geschützt zu werden, und dem Recht des Einzelnen auf körperliche Unversehrtheit.

Neben dem Grundrecht auf körperliche Unversehrtheit schützt die Menschenwürde aus Art. 1 Abs. 1 S. 1 GG als verfassungsrechtliches Grundprinzip davor, dass der Mensch nicht zum Objekt staatlichen Handelns gemacht wird [BVerfGE 88, 203 (251f)]. Eine generelle Impfpflicht mit einem neuen Impfstoff birgt aufgrund der noch unerforschten gesundheitlichen Folgen das Potenzial, den Menschen zu einem solchen Objekt zu machen, indem durch eine absolute Impfpflicht die Entscheidung abgenommen wird, ob ein solcher Eingriff in die körperliche Integrität erwünscht ist [4]. Im Rahmen der Grundrechtsabwägung ist dabei nicht nur auf rein medizinische und epidemiologische Erkenntnisse und Effizienz abzustellen, sondern eine ausreichende Berücksichtigung der Grundrechte des Einzelnen vorzunehmen [5]. Medizinisches Fachwissen und neuste Forschungserkenntnisse sind daher im Rahmen des Verhältnismäßigkeitsgrundsatzes wohl nur als ein Kriterium der Geeignetheit und Erforderlichkeit einer etwaigen Maßnahme heranzuziehen [6].

Gleichwohl hat das Bundesverwaltungsgericht bereits am 14.07.1959 entschieden [BVerwG Urt. v. 14.07.1959 I C 170.56.], dass eine Impfpflicht dann mit dem Grundgesetz vereinbar ist, wenn es sich um besonders ansteckende Krankheiten handelt, die Leben und Gesundheit anderer Menschen schwerwiegend gefährden. In einem 
solchen Fall ist der Schutz der Gesundheit anderer Personen und der Allgemeinheit zur Abwehr einer potenziellen Seuchengefahr ein verfassungsrechtlicher Rechtfertigungsgrund für den Eingriff in das Grundrecht auf körperliche Unversehrtheit. Demgegenüber sind nach dem BVerfG amtsärztliche Zwangsuntersuchungen hinsichtlich der körperlichen Unversehrtheit dann unzulässig, wenn bei relativ unwichtigen staatlichen Anliegen schwerwiegende Untersuchungsrisiken in Kauf genommen werden müssten [BVerfGE 16, 194 - Liquorentnahme]. Um somit eine Prognose über die Verfassungskonformität einer potenziellen Impfplicht abgeben zu können, bleiben nach Zulassung der ersten Impfstoffe gegen das SARS-CoV-2-Virus die daraus resultierenden gesetzlichen Regelungen abzuwarten.

\section{KERNAUSSAGEN}

- Nach §20 Abs. 6 IfSG ist eine Schutzimpfung für bedrohte Teile der Bevölkerung durch Rechtsverordnung in Deutschland zulässig, wenn eine übertragbare Krankheit mit klinisch schweren Verlaufsformen auftritt und mit ihrer epidemischen Verbreitung zu rechnen ist.

- Eine Impfpflicht bedarf jedoch der grundrechtlichen Abwägung im Rahmen des Art. 2 Abs. 2 GG.

- Abzuwarten ist, ob das BVerfG auch zukünftig verpflichtende Schutzimpfungen für zulässig erklärt, indem es davon ausgeht, dass Schutzimpfungen nicht nur das Individuum gegen die Erkrankung schützen, sondern gleichzeitig die Weiterverbreitung der Krankheit in der Bevölkerung verhindern.

- So sollen auch Personen geschützt werden können, die aus medizinischen Gründen selbst nicht geimpft werden können, bei denen aber schwere klinische Verläufe bei einer Infektion drohen.

\section{Interessenkonflikt}

Die Autorinnen/Autoren geben an, dass kein Interessenkonflikt besteht.

\section{Autorinnen/Autoren}

Dr. jur. Dr. rer. hum. biol. Fabian-S. Frielitz,
LL.M., M.A., MBA, MHEd
Seit 2015 wissenschaftlicher Koordinator des
Zentrums für Bevölkerungsmedizin und Ver-
sorgungsforschung (ZBV) und wissenschaftli-
cher Mitarbeiter am Institut für Sozialmedizin
und Epidemiologie (Universität zu Lübeck). SP:
Gesundheitsökonomie und Digitalisierung.

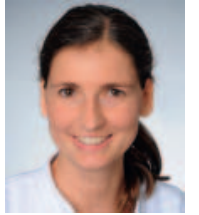

\section{Dr. med. Isabel Viola Wagner}

Seit 2018 Fachärztin für Kinder- und Jugendmedizin. Aktuell in der Weiterbildung zur Pädiatrischen Endokrinologin. SP: Auswirkung metabolischer Erkrankungen auf die Hodenfunktion und Fertilität.

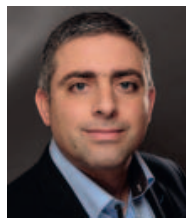

\section{Priv.-Doz. Dr. med. Denis Martin Schewe}

Seit 2011 in Kiel mit Abschluss der Weiterbildung im Bereich Kinderhämatologie/Onkologie. Seit 2015 Oberarzt und Leiter einer AG zur translationalen Leukämieforschung. Weiterer SP: telemedizinische Versorgung onkologisch erkrankter Kinder und Jugendlicher.

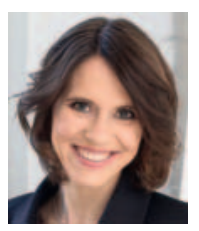

Dr. jur. Klara Bothe, LL.M.

Doktorratsstudium im europäischen Arbeitsund Sozialrecht an der Universität Wien und aktuell (noch) Rechtsreferendarin bei der Freien Hansestadt Hamburg.

Korrespondenzadresse

Dr. jur. Dr. hum. biol. Fabian-S. Frielitz Institut für Sozialmedizin und Epidemiologie Universität zu Lübeck Ratzeburger Allee 160 23562 Lübeck Deutschland fabian.frielitz@uksh.de

\section{Literatur}

[1] Infektionsschutzgesetz vom 20.07.2000, BGBI. I S. 2652.

[2] Drittes Gesetz zum Schutz der Bevölkerung bei epidemischen Lagen von nationaler Tragweite vom 18.11.2020, Nr. 52, BGBI. I S. 2397.

[3] Gesetz für den Schutz vor Masern und zur Stärkung der Impfprävention (Masernschutzgesetz) 2020 Feb 13; BGBI. I S. 148.

[4] BVerfG Beschluss vom 11.05.2020, 1 BvR 469/20, 1 BvR 470/20.

[5] Vgl. zu den sozialen Folgen auch: Wissenschaftlicher Dienst des Deutschen Bundestages, Kontaktbeschränkungen zwecks Infektionsschutz: Grundrechte, WD 3 - 3000 - 079/20, Berlin 2020; $37 f$

[6] Engels A. Infektionsschutzrecht als Gefahrenabwehrrecht? Die Öffentliche Verwaltung (DÖV). 2014: 464-472

[7] BVerwG Urt. v. 14.07.1959 - I C 170.56.

\section{Bibliografie}

Dtsch Med Wochenschr 2021; 146: 206-208

Online-Publikation: 13.1.2021

DOI 10.1055/a-1332-9841

ISSN 0012-0472

(C) 2021. Thieme. All rights reserved. Georg Thieme Verlag KG, Rüdigerstraße 14, 70469 Stuttgart, Germany 